\title{
Drills as Tools for Media Penetration and Sampling
}

Yoseph Bar-Cohen and Kris Zacny

\section{1}

\section{Introduction and Historical Perspective}

Drilling and excavation are widely applied for many purposes, including making a hole in a wall, deep drilling in the search for oil and exploration of the Earth's subsurface (Clark, 1987). Most of what we have learned about early climates of planet Earth (e.g., ice ages) was obtained from cores taken by drilling through ice sheets and glaciers (e.g., Dansgaard, 2004). Increasingly, developers of drills are addressing complex challenges in extreme environments, for example penetrating the surface of planetary bodies. Drilling on the Moon is an example of such a challenge, which was successfully accomplished for the first time in 1970 by the Soviet's robotic Luna 16 lander. This success was followed with the growing efforts to drill on Mars and penetrating the surfaces of other extraterrestrial bodies and increasingly enabling scientists to investigate the history of our Solar System and understand better our own planet Earth (Zacny et al., 2008). To address the challenges presented by the large variety of materials that need to be penetrated in drilling into our own planet's surface, scientists and engineers have developed many types of drills, with mechanical drills being the most common. Mechanical drills use a bit with a tip that interacts with the drilled medium and applies cutting or breaking forces over a small area to achieve large shear and/or impact stresses. These types of bits are widely used and can be purchased at local hardware stores.

Penetrating solid objects, such as the ground, rocks and wood, has been achieved by creatures and plants in Nature ever since they first existed on Earth millions of years ago. The earthworm, termites (Figure 1.1), rodents, the woodpecker (Figure 1.2) and many others are capable of making holes and tunnels for their habitat and search for food (Bar-Cohen, 2005). Also, roots of plants have amazing capabilities to penetrate rocks and hard soil.

Since ancient times, humans have been digging through the ground and solid objects. There are many reasons to dig and, over the years, as more effective tools became available, the capability to penetrate various media increased. Some of the 


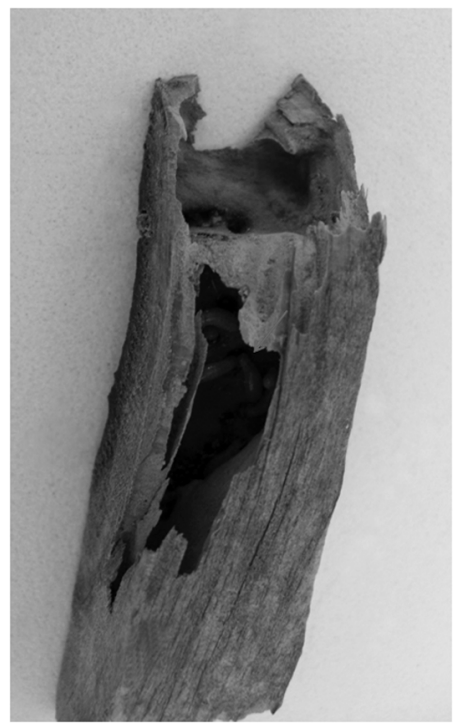

Figure 1.1 Termites (see inside the top section of the cavity) make holes in wood.

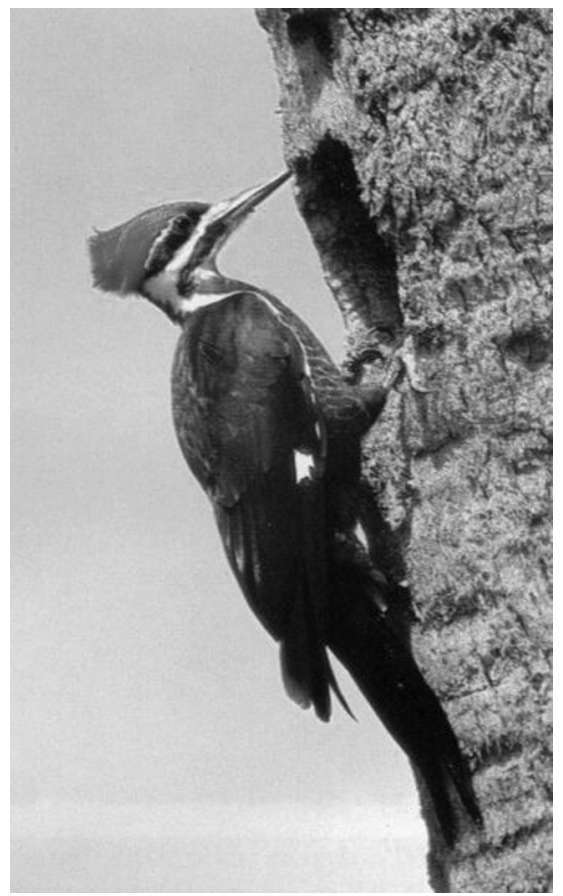

Figure 1.2 The pileated woodpecker performs "percussive" drilling to bore holes in trees to make their nest and also seek food. Courtesy Paul and Bernice Noll http://www.paulnoll.com/ Oregon/Birds/habitat-Woodpecker.html. 
applications that required penetration of the ground included mining for resources, digging water wells, burying objects, supporting columns and structures and searching for food (including plant bulbs and roots). Advances in penetration tools were made as a result of discovering more effective fabrication materials, developing methods of processing and machining, increasing the capability to leverage forces and driving tools with the aid of mechanical, electrical, pneumatic and hydraulic actuators.

The use of metallic tools for the penetration of objects probably started in the Bronze Age when tools were made in the shape of an arrow that consisted of two distinct cutting edges. The use of bow drills dates back to the ancient Egyptians (3150-31 BC). As far back as 2550-2315 BC, the Egyptians may have used diamond drilling tools for the construction of the pyramids, and between 600 and $260 \mathrm{BC}$, the Chinese drilled holes up to $35 \mathrm{~cm}$ (14 in) in diameter to depths exceeding $600 \mathrm{~m}$ ( $2000 \mathrm{ft}$ ). About 1000 years ago, in $1126 \mathrm{AD}$, Carthusian monks used a percussive technique to drill for water, reaching a depth of around $300 \mathrm{~m}$ (1000 ft) (De Villiers, 2001).

The development of pneumatic drills dates back to Samuel Ingersoll's invention in 1871 and it made a major impact on the ability to drill. The electric drills in the past century have revolutionized our ability to penetrate tough materials on demand. The invention of the steam engine in the eighteenth century had an enormous impact on the capability to drill on large scales and with it came a surge in demand for coal to fuel steam engines. One of the most extracted materials is coal, which is also the most abundant fossil fuel on Earth: its predominant use has been for producing energy in the form of heat. In the eighteenth and nineteenth centuries, it was the most important energy source that fueled the Industrial Revolution and was a major driver in the development of tools for large-scale applications. The capability to mine hard rocks and the reduction in the cost and time required for excavation were a direct result of using mechanical drills powered by compressed air, that is, the pneumatic hammers that are known today as "jackhammers".

A rotary steam-driven drill was invented by Richard Trevithick in England in 1813 [Britannica on-line] and the first patented pneumatic drill was invented in 1849 by Jonathan Couch in Philadelphia, PA, USA (Encyclopedia Britannica, 1986). This pneumatic drill was a hammer-type drill that impacted a metal rod into rocks. This particular drill and many others, invented by Joseph W. Fowle (1849-1851) and Cavé in Paris (1851), were driven by steam. Although these drills found numerous applications on the surface, they were not suitable for underground drilling, where in fact most of the drilling for mineral excavation was taking place. In the early stages, the steam machinery had to be kept close to the boiler, since lengthy hoses were not available and the steam lost its heat and pressure when transported over long distances. In 1851, Fowle patented the first use of a flexible steam hose and thus made the location at which the drills are positioned less dependent on the location of the boiler. Thus, Fowle's patent provided the basis for the design and development of modern rock drills.

In 1852, the use of compressed air for rock drilling for the creation of the Mont Cenis tunnel in the Western Alps was proposed by the physicist Colladon. The idea of using compressed air to drive the drilling tools was also utilized by the Italian 
engineer Germain Sommeiller and others between 1852 and 1860 (Peele, 1920) to develop new types of drills. The use of compressed air offered three major advantages that included:

1. Low transmission losses.

2. No safety issues related to leakage (as opposed to the case of steam, which posed a burn hazard to the operators when leaks occurred in the pipes).

3. The "used" compressed gas provided additional ventilation in the working area (e.g., a mine shaft or a tunnel).

A notable advancement of the pneumatic drill was made in 1890 by C. H. Shaw [Britannica on-line], a machinist from Denver, CO, USA, who invented the first hammering drill with air-leg feed (Mining Magazine, 2006). A significant improvement in pneumatic drilling was made in an 1896 invention by the entrepreneur J. George Leyner from Colorado, who introduced the hollow drill bit. In this arrangement, compressed air was blown down the center of the drill and out through the hollow drill bit. The air not only removed the cuttings from the hole, which improved the drilling efficiency, but also helped to keep the drill bit and the drilled formation cool. Drilling hazards in underground mines were very common. Drillers suffered respiratory illnesses due to breathing the released fine dust generated by drilling rocks. This problem of dust was substantially reduced by J. George Leyner, who suppressed dust formation by introducing water into the drilled holes. The application of diamonds as cutting elements in drill bits was first proposed in 1869 . Although diamond bits were (and still are) much more expensive than other types of bits, they provided much greater durability in cutting hard materials and this made the drilling process more effective and efficient.

The ever-increasing demand for resources led to the essentially complete exploitation of easy-to-mine areas and left more difficult areas (such as deeper mines or very remote mines in the cold Arctic regions) for the future. The gold mining industry now has the deepest mines in the world. For example, the AngloGold Ashanti gold mine Tau Tona (a Setswana word for Great Lion) outside Carletonville, South Africa, is $3.6 \mathrm{~km}$ deep (Figure 1.3). The mountains of tailings in the foreground of this figure are processed (crushed to powder and chemically treated) gold-bearing rock recovered from the mine. A great deal of drilling and blasting was carried out to produce these large hills. Currently, there are plans to reach as deep as $3.9 \mathrm{~km}$, where more gold-bearing rocks can be found. At these great depths, rock temperatures reach $55^{\circ} \mathrm{C}$ and extensive air conditioning is required for the miners in order to cool the air temperature to the bearable level of $28^{\circ} \mathrm{C}$. The challenges of drilling to great depth under harsher conditions together with tighter economic considerations that include mining faster are increasingly raising the required capabilities of cutting tools. Therefore, there is a continuing need to improve the performance of cutting materials used at the tips of the drill bits in order to penetrate harder formations more quickly and with much greater durability.

The development of metallurgical processes to heat-treat steels for greater deformation resistance of drill rods dates back to the $1890 \mathrm{~s}$. This initial development led to improved hammer drills that were faster, lighter and significantly more effective. 


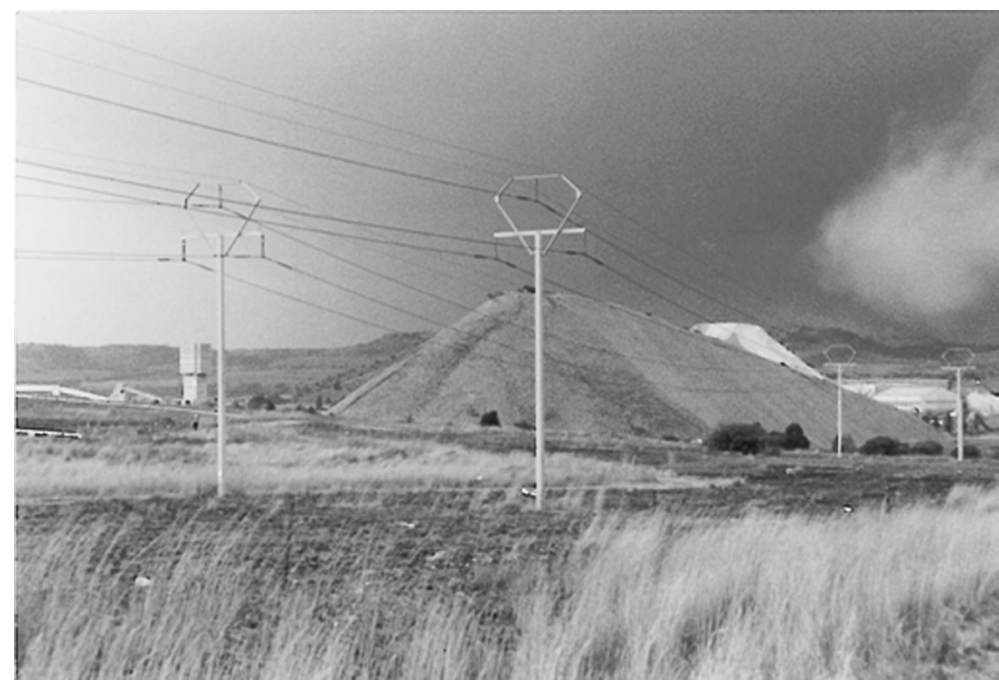

Figure 1.3 Tau Tona gold mine in South Africa, at $3.6 \mathrm{~km}$ deep, is the world deepest mine (the shaft is visible in the background). The hills in the foreground are tailings of recovered and processed gold-bearing rock.

Today, drill rods can slide more freely in a chuck and do not deform when the piston hits their top end. The invention of the replaceable bit in 1918 by A. L. Hawkesworth, a mechanical foreman for the Anaconda Company in Butte, MT, USA, made drilling even more efficient, because now only cutting edges were replaced and not entire drill sections. After World War II, hand-held jackhammers were developed that could be flexibly attached to an air cylinder; these drills allowed miners to drill in any desired direction without the need to mount the drill on a support structure. The speed of drilling has subsequently increased and, in addition, new bits made of much harder materials such as tungsten carbide were produced. In the 1970s, hydraulic technology was applied to drive drills directly instead of being used to create the compressed air that powered the drills. Advances in technology of hydraulic drills led

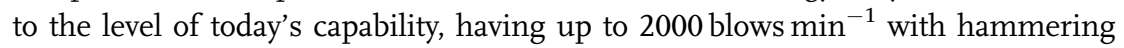
action that is much quieter than previously, with noise levels as low as $85 \mathrm{~dB}$ at a distance of $10 \mathrm{~m}$ (e.g., Altras Copco SmartRig) (Mining Magazine, 2006).

The invention of the electric rotary drill is credited to the scientist James Arnot, who developed this type of drill in 1889 while an employee of the Union Electric Company in Melbourne, Australia. He designed it primarily to penetrate rocks and coal. Six years later, the invention of the portable drill was patented by Wilhelm Fein in Germany. Another major milestone occurred in 1917, when Black \& Dekker invented the trigger switch that was mounted on a pistol-grip handle of the drill (Decker and Black, 1917). Modern oilfield rotary drills were first introduced in 1884 (Maurer, 1968); but the first oil well was drilled in 1745 in France (Lee et al., 1988). 


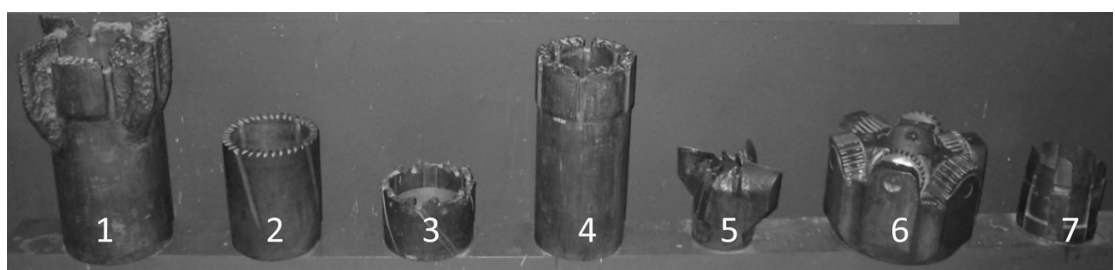

Figure 1.4 Assortment of bits from the middle of the twentieth century displayed at the Deutsches Museum in Munich, Germany. This photograph was taken by Jack Craft, Honeybee Robotics Spacecraft Mechanisms Corporation, and it is published courtesy of him. The description of each of these drill bits was translated from German by Jack Craft and it is provided with editing in the main text.

In Figure 1.4, an example is shown of an assortment of bits from the middle of the twentieth century that is displayed at the Deutsches Museum in Munich, Germany. These coring bits were designed for cutting cores out of rock. They consist of a cylindrical steel body and a cutting edge having a shape that is specifically designed for the specific rock formation hardness of rotary drills. Nowadays, diamonds are almost exclusively used at the cutting edges since they provide higher penetration rates and longer drill bit life than metal edges. The following is the list of drill bits that are shown numbered in Figure 1.4:

1. This coring bit was used for soft rock formations and its main advantage was that the cutting structure could be restored to the original shape by merely replacing the hardened metal on the worn-out teeth. Note the four cutters that are perpendicular to the circumference of the core drill and four additional cutters that are placed along the circumference of the core drill. The function of the latter cutters was to cut out the core, whereas the former cutters were designed to excavate the annular area and to sweep the cuttings produced to the outside.

2. This bit was designed for medium-to-hard formations. It had hardened steel teeth and slanted drilling fluid channels (also called "junk" slots) on its outside surface to allow for easy removal of drilled cuttings.

3. This drill bit was also designed for penetrating medium-to-hard formations. This bit could be restored to its original shape by replacing the hardened metal on the worn-out teeth.

4. This bit was designed for hard formations. It is a simple core-drill bit with hardened metal cutting edges.

5. This bit is only suitable for soft formations and it has two sets of "wings". The four inner ones are actually cutting teeth that drilled the rock, whereas the four outer large wings swept the cuttings to the outside and in turn helped to keep the bottom of the hole clean. 
6. Unlike the previous five drill bits that used fixed cutters, this drill bit is a roller cone bit, whereby cutters are placed on rotating "wheels" around the rim of the drill bit. As the drill bit rotates, these six rollers also rotate in unison. The cutting takes place essentially by crushing the rock underneath from a crushing load imposed by the cutter structure. This is a different drilling mode, since the previous five cutters in fact cut or sheared the rock surface rather than crushed it. These types of bits were used for medium-hard to hard formations, depending on the type of roller teeth used.

7. This device is not a drill bit but, rather, a core catcher that is inserted into the bit prior to coring operation. It consists of a number of thin plates or wings. Note that unlike all the drill bits, which are shown upside down, this part is shown in its correct vertical operation direction. As the drill is being pulled out of the hole, the wings of the core-catcher wedge into the surface of the core. This action not only helps to break the core off the bottom by developing tensile stresses in the core, but also helps to retain the core inside the coring bit.

The advent of battery technology contributed significantly to the portability of drills and it enabled the development of the first cordless electric drill, which was introduced by Black \& Decker in 1961. Cordless technology allowed the drilling by the Apollo 15, 16 and 17 astronauts to depths of more than $70 \mathrm{~cm}$ (achieved on earlier missions by hammering in core tubes). Between 1971 and 1972, three Apollo lunar missions used a battery-operated rotary-percussive drill to acquire cores and implant sensors into the surface of the Moon (Decker and Black, 1961). The drill, called the Apollo Lunar Surface Drill, was used to produce holes for the deployment of heat probes for measuring of heat flow out of the lunar surface and also for collecting a continuous core of lunar regolith. The Apollo 15, 16 and 17 cores of lunar regolith brought back from the Moon (Heiken and Jones, 2007) provided important material for scientific study of the Moon.

Drilling on the Moon was a major challenge and drilling the deepest hole on Earth was even more so. Although the original idea of drilling a hole on Earth with a depth target of $15000 \mathrm{~m}$ (49210 ft) was first proposed in the former Soviet Union in 1962, the actual drilling project did not commence until 1970, after more than 8 years of preparation. The Kola Peninsula in the northwest Soviet Union (Figure 1.5) was chosen as the best location for this project, dubbed the Kola Superdeep Borehole project. The objective was purely scientific - to learn about the properties of subsurface rocks - and the science payoff was indeed outstanding. To this day, the cores acquired from these great depths continue to be analyzed and are a source of many scientific publications. In addition to discovering that below the 3-6 km granite layer there is a metamorphic layer rather than the basalt rock layer that was previously supposed, scientists found microscopic fossils as deep as $6.7 \mathrm{~km}$ below the group surface. In 1989, the drill reached its greatest depth of $12261 \mathrm{~m}(40226 \mathrm{ft})$ and analysis of recovered rock samples has shown them to be 2.7 billion years old (Kola, 1989). Unfortunately, using this drill to reach greater depths was not feasible because of the very high temperatures encountered, in the region of $180^{\circ} \mathrm{C}\left(356^{\circ} \mathrm{F}\right)$, which made the rock more plastic and in turn more difficult to drill. Drilling to these 


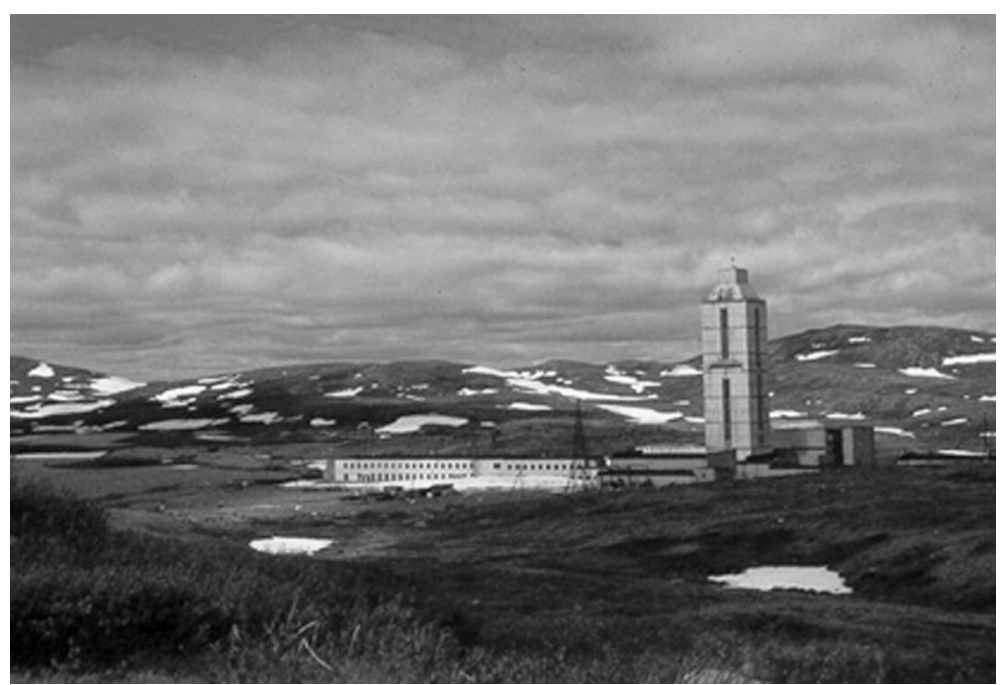

Figure 1.5 The Kola-SG3 drill-rig enclosure and location of the Kola Superdeep Borehole project which drilled $12262 \mathrm{~m}$ (7.5 miles) into the Earth's crust (based on June 2000, K.C. Schulze, http://www.geo.uni-bonn.de/members/schulze/).

great depths required drilling engineers to invent ever newer drilling technologies. This project still remains one of the greatest drilling achievements ever. In 2008, efforts to dismantle this facility have started to bring this project to its end.

In the USA, a parallel initiative to conduct ultra-deep drilling was taken in 1961. This project, called Mohole, was led by the American Miscellaneous Society and was funded by the National Science Foundation (NSF) (Burleson, 1998). Its goal was to drill a hole through the ocean floor to reach the boundary between the Earth's crust and the mantle. Drilling was done through $3500 \mathrm{~m}(11700 \mathrm{ft})$ of water and then through the seafloor to a depth of $183 \mathrm{~m}$ ( $601 \mathrm{ft}$ ) off the coast of Guadalupe, Mexico. Unfortunately, the project was canceled by US Congress in 1967.

The hunger for oil led to the emergence of drilling in a non-vertical direction or at a slant, also known as directional drilling. The technology was used as early as the 1920 s, where rigs on one property were used to tap into reservoirs in neighboring properties. A key issue related to this technology is the ability to track the drilling direction in relation to the geological area that is being drilled.

Another challenging area for drilling is subsurface penetration of extraterrestrial planetary bodies in order to acquire extraterrestrial samples for detailed analysis. The acquisition of samples from the subsurface of extraterrestrial bodies involves challenges that are far more complex and demanding than similar operations on Earth because of the limitations on mass, power and human control (Further details can be seen in Chapters 7 and 8). On Mars it will be necessary to drill deeper than a few meters to reach locations where oxidation and irradiation by cosmic rays have been minimal, thus suggesting that the possibility of finding evidence of putative extraterrestrial life may be higher. Subsurface samples can also provide evidence of 
past climates and geological events. The acquired samples can either be analyzed on the surface using a suite of in situ instruments or, ideally, brought back to Earth for a more thorough analysis using more sophisticated equipment.

Extraterrestrial drill systems have to be able to penetrate unknown media (this is referred to as geological uncertainty), which could be rocks of various degrees of hardness, compacted soil, pure ice, dirty ice or even frozen carbohydrates as is the case on the Saturn's moon, Titan. Also, a drill system has to be able to operate successfully in dusty environments that may be cold or hot and where the pressure may be high, low or a vacuum. For example, the pressure on the surface of Venus is over 90 times higher than the Earth's atmospheric pressure (or as high as at a depth of $1 \mathrm{~km}$ in the Earth's oceans) and the surface temperature can be as high as $480^{\circ} \mathrm{C}$. Another planet with an extreme environment is our Moon, which has no atmosphere and where the surface temperature can vary from $-233^{\circ} \mathrm{C}$ at night in the polar regions to $+123^{\circ} \mathrm{C}$ during the day in the equatorial regions. In addition, the surface that faces the Sun can be significantly hotter than the surface that is facing deep space. The resulting high temperature gradient needs to be addressed to make sure the drilling system can survive these potentially damaging conditions. In addition, planetary missions have strong constraints on the mass and power that can be available. Another factor that affects the operation of drills is the local gravity which provides the necessary capability to pre-load the drill from its support platform (rover or lander). For Mars the gravity is about one-third of that on Earth, and for asteroids and comets local gravity may be many hundreds or thousands times smaller.

Operational autonomy is an obvious requirement for drill systems deployed on the other planetary bodies. The communication delays that approach 20 min each way make remote control operation impractical on Mars. Once a command is sent out, it takes more than 40 min to learn whether the drill indeed started to drill or not and the presence of any problems would be indicated to the operators on Earth only about 20 min after they had occurred.

\section{2}

\section{Methods of Drilling and Penetration of Objects}

In general, there are three basic approaches to breaking rocks: mechanical, thermal (thermal spalling, thermal melting and vaporization) and chemical approaches. The focus of this book is on methods of drilling that use mechanical forces to penetrate objects. However, for the sake of completeness, this section provides an overview of the various alternative drilling techniques.

\subsection{1}

\section{Mechanical Techniques}

The most widely used techniques for drilling are based on mechanical methods that apply stresses to exceed the rock tensile or shear strength, causing brittle failure or plastic yielding. In rotary drilling, the most widely used method, a cutter cuts or 


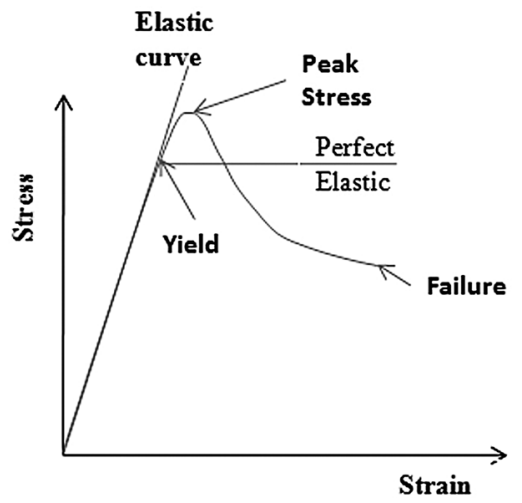

Figure 1.6 Typical stress-strain curve of a rock under loading.

shears the drilled rock. On the other hand, in the case of percussive techniques, repeated impacts produce finely crushed rock directly underneath the impact location. At high impact stress levels, cracks are initiated around the crushed zone, creating rock fragments (Maurer, 1968).

Under mechanical loading, most rocks behave as elasto-plastic materials, exhibiting strain hardening and softening (Further details can be seen in Chapter 2). A typical stress-strain curve of a rock under axial stress is shown in Figure 1.6. Subjecting a rock to a stress level that is higher than its yield stress causes a permanent deformation. If the rock behaves as a brittle material, it will fracture, and if it is more plastic, it will deform. Rocks can also fail in fatigue; cyclic loading can lead to the formation of miniature cracks that grow with time until they reach critical lengths, at which point the rock breaks.

Rocks can be subjected to tensile, shear and pure compressive stresses. Tension and shear stresses cause failure of the intergranular bonds, whereas pure compression stresses lead to crushing and breakage of grains and shear failure. At different locations within the rock material that surrounds the drill bit, the drilling process may introduce all three types of stresses. Examples of the analytically determined principal strain field at various times after impact from solid and coring bits are shown in Figure 1.7. Generally, the relative levels of tension, shear and compressive stresses are influenced not only by the loading mode (compressive impact and/or frictional shear loading), but also by the bit and borehole geometry and by dynamic stress wave reflections that are generated by the drilled medium.

Challenges increase when drilling deep into the subsurface. If the formation consists of soil or regolith, the wall of the borehole that is formed needs to be encased in order to avoid collapse. Pressure from the borehole wall grows as the drilling depth increases and requires adequate attention during drilling. If the medium that is drilled consists of ice, refreezing of the melted water can jam the bit.

\subsubsection{Erosion via Water Jets or Particles Blast}

Erosion via water jets and particle blasts are effective means of penetrating various media. Using a jet of abrasive particles at flow rates of $10-100 \mathrm{~m} \mathrm{~s}^{-1}$ creates an 


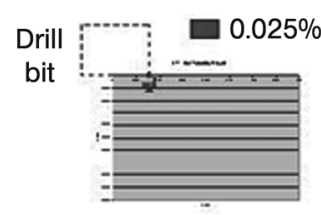

$36 \mu \mathrm{s}$

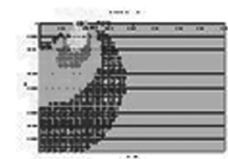

$46 \mu \mathrm{s}$

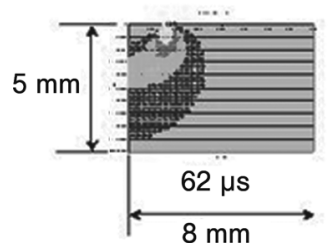

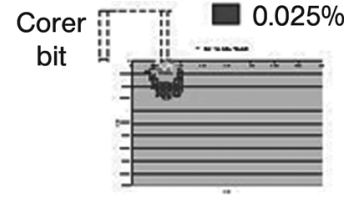

$36 \mu \mathrm{s}$

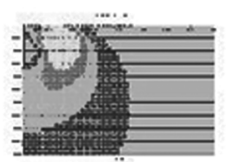

$46 \mu \mathrm{s}$

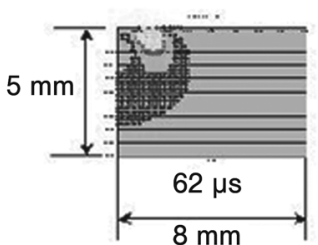

Figure 1.7 The principal strain profile at various times after impact of a drilling bit and a coring bit (Bao et al., 2003). (Please find a color version of this figure on the color plates.)

erosion capability that is sufficient to penetrate rocks (Maurer, 1968). Penetration of rocks using pressurized water jets requires much higher flow rates of 200-1000 $\mathrm{m} \mathrm{s}^{-1}$. This technique is so effective that it can even be used to cut through metals with relatively high precision. The rock fracture process is a result of an intense pressure gradient that is formed by the water jet. Another benefit of using water jets for drilling is that the resultant cuttings are removed by the water jet.

\subsubsection{Explosive Excavation}

Explosive excavation requires a relatively small amount of energy, instantaneously unleashes significant forces and is a quick method of excavating rocks. A detonator is used to trigger the explosion and the amount of explosives and their location determine the extent of material that is fractured. This method can be used to remove very hard materials but it is very difficult to control and failures can have severe consequences. Generally, explosive excavation has been in use for mining since around the late 1500 s and was used in construction industry to create new roads in rural areas as early as 1675 [http://www.explosives.org/HistoryofExplosives.htm].

To reach depths at the level of kilometers, Russian workers (Ostrovskii, 1960; Wyllie, 1999) used an explosive drill that consisted of a capsule inside a tool. The gases created during the explosion were channeled through a nozzle and impacted the bottom of the hole, leading to drilling rates that were as high as $15 \mathrm{~m} \mathrm{~h}^{-1}$. However, the nozzle had to be maintained at a vertical distance of about $30 \mathrm{~cm}$ from the bottom of the hole. As the drilling progressed, a fluid-type explosive was fed continuously into the combustion chamber above the nozzle and, in order to operate continuously, a flushing fluid had to be used. 
For underwater excavation applications, an explosion spark discharge technique has been developed. A high-power electric field ionized gas in a condenser produces high-temperature plasma, which leads to the formation of a high-pressure pulse in the range of hundreds to thousands of atmospheres. This burst is very effective in water since water is essentially an incompressible medium and the relatively high "explosive" power made this technique very useful also as an underwater seismic source (Maurer, 1968).

\section{2 .2}

\section{Thermal Techniques}

There are two principal thermal methods: thermal spalling and thermal melting. The difference between these two techniques is that thermal spalling occurs at lower temperatures of about $400-600^{\circ} \mathrm{C}$ whereas thermal melting followed by vaporization requires temperatures that are, typically, in the range $1100-2200^{\circ} \mathrm{C}$ (Maurer, 1980).

\subsubsection{Thermal Spalling}

Thermal stresses that are generated by applying heat are created by the mismatch in thermal expansion of the constituents and grains within the structure of rocks (Just, 1963). This process is called thermal spalling and it causes fracturing and degradation of rocks that result in flaking of rock fragments. Its effectiveness depends on the thermal gradients produced in the rock. The method is limited in its applicability since not all rocks are sufficiently heterogeneous to sustain spalling.

Rock breakage that results from thermal spalling is a natural process and is referred to as "onion weathering" or "exfoliation". In deserts, rock surfaces heat up and expand during the day and cool and contract during the night. This cycle of expansion and contraction creates small cracks that grow with time until entire layers of rock peel apart and fall off. The process can be accelerated if water is present in the cracks; when water turns into ice, it expands in volume and forces the surfaces to separate.

Most rocks suffer degradation and lose of strength when heated and can reach a point of being easy to crumble or break - particularly if a rock is heated nonuniformly. Heat can be applied by laser and the degraded rock material can be removed using techniques that may include high-voltage sparks or water-jet erosion.

\subsubsection{Melting and Vaporization}

Exposing a rock to very high temperatures induces melting and vaporization of the rock material. For example, a controlled-shape hole can be formed using a highintensity laser beam (Ready, 1997). The disadvantage of this technique is the very high power that is required to melt a rock. This process, however, can be readily applied to melt ice. Ice melting drills are frequently used in the Antarctic to drill holes in ice sheets and ice shelves. Penetration rates as fast as $1 \mathrm{~km}$ in $10 \mathrm{~h}$ is not uncommon. Ice melting drills have also been proposed for drilling on Europa, a moon of Jupiter, whose surface is composed of water-ice, and on Mars, which has 


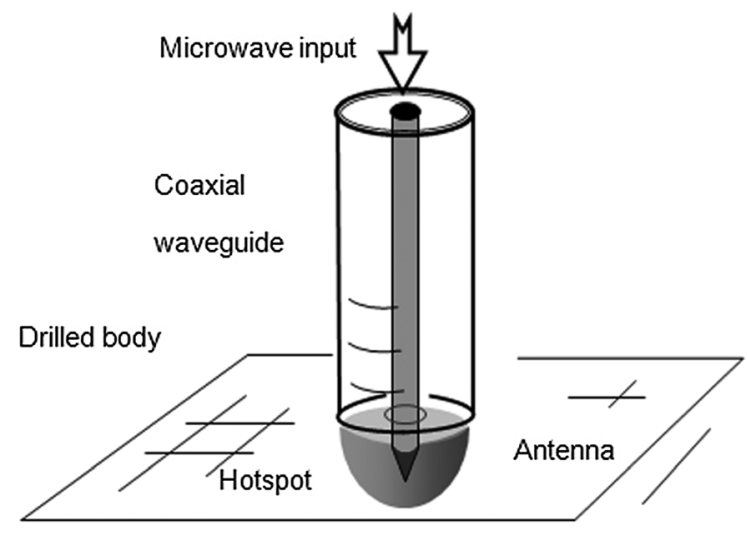

Figure 1.8 A schematic illustration of the microwave drill. Courtesy Eli Jerby, Tel Aviv University, Ramat Aviv, Israel.

a water ice cap at its north pole and has considerable embedded ice in the subsurface regolith at high latitudes (Smith et al., 2006; Rapp, 2007; Chapter 4 of this book).

Microwave drilling is another method that uses heat to penetrate objects. The microwaves can be applied directly to the medium by inducing heat resulting from dielectric losses (Maurer, 1968) or via a heated bit-like element. A method based on the latter approach was developed by researchers at Tel Aviv University, Israel (Jerby et al., 2002) and the drill was designed as a coaxial near-field radiator that is fed by a conventional microwave source (Figure 1.8). The center electrode of a coaxial radiator acts as the bit. The drill bit serves as an antenna to focus microwave energy on to a small spot under the surface of the drilled material, thereby melting any non-conductive materials along its path. This method can create temperatures as high as $1500^{\circ} \mathrm{C}$ and a prototype device, using a $2 \mathrm{~mm}$ diameter bit, drilled a $2 \mathrm{~cm}$ deep hole in concrete in about $1 \mathrm{~min}$. In another test that used mechanical means to remove the debris produced during drilling, a $12 \mathrm{~mm}$ diameter, $10 \mathrm{~cm}$ deep hole in concrete was produced (Figure 1.9). The advantages of this method are that it does not require a rapidly rotating bit and it does not produce dust or noise. Although its operation as a quiet device that does not produce powder makes a microwave drill advantageous for operating under conditions such as construction in urban areas at night or even military tasks, it is not applicable for in situ planetary exploration due to the high power that is required and the thermal damage incurred by the drilled material.

\section{2 .3}

\section{Chemical Techniques}

There are various chemicals, including fluorine and halogens, which can be used to dissolve rocks and other solids (Maurer, 1968) and thus penetrate the subsurface of 


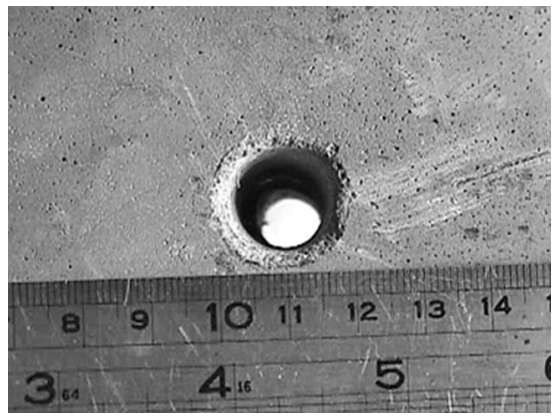

Figure 1.9 A $12 \mathrm{~mm}$ diameter, $10 \mathrm{~cm}$ deep hole in a concrete slab produced by a microwave drill that was mechanically assisted for debris removal. Courtesy Eli Jerby, Tel Aviv University, Ramat Aviv, Israel.

such solid media. These techniques can be fairly effective in penetrating specific types of rocks (McGee, 1955). However, the process of penetration involves a violent reaction and may even cause fire in addition to posing health hazards to its users.

\section{3}

\section{Types of Mechanical Drills}

There are many types of mechanical drills that are powered manually, electrically, hydraulically or pneumatically. Their sizes vary from hand-held models to giant rigs such as those used in the oil industry. Portable drills are increasingly being designed as cordless types using rechargeable batteries, which provide similar functions to the drills that run on electrical AC power (e.g., 110 or $220 \mathrm{~V}$ ) or pneumatic power. Portable drills are also available as hammer drills but most of them are used for the convenience of drilling small holes in a wall and for driving screws.

\subsection{1}

\section{Rotary Drill}

Rotary drills are the most widely used drills. They include both portable and fixture mountable versions. The latter is also known as a drill press, a pedestal drill, a pillar drill or a bench drill and it is mounted on a stand or bolted to the floor or workbench. A drill press offers various advantages over hand-held drills, particularly in requiring less effort to apply force onto the drilled medium and to control more precisely the position and linearity of the drilled hole. The movement of the chuck and spindle in the press drill is accomplished by a lever that provides the operator with considerable mechanical advantage. The drill consists of a base, column (or pillar), table, spindle (or quill) and drill head and it is typically driven by an electric motor. 


\subsection{2}

Hammer Drill

Hammer drills use impact action at various frequencies to penetrate objects and are widely used to penetrate brittle materials, such as concrete. Large hammer drills, especially the pneumatically driven ones, are crude in their action and the energy delivered in each stroke is highly variable. The cheaper drills are used to smash the drilled object and they introduce significant vibrations into the surroundings that can cause collateral damage. The Special Direct System (SDS) type of drill, which was developed in the 1970s by Bosch, produces a high-power hammering action that can gently pulverize the drilled material with less damage to the borehole.

\subsection{3}

\section{Rotary-Hammer Drill}

A rotary-hammer drill, also known as roto-hammer or masonry drill, is effective in drilling hard concrete and masonry. This type of drill operates percussively on rocks or concrete and applies its weight to introduce impact forces while also rotating the bit to remove the cuttings. As mentioned earlier, the combined action significantly enhances the operation of the drill over the use of either rotation or hammering only.

\section{4}

\section{Bits - the End-Effector of Drills}

A drill bit is the end-effector of a drilling device that makes the borehole needed in the desired medium and it is driven by an actuator that is mounted in the body of the drilling device. The drill bit determines the diameter of the borehole formed and the characteristics of the sample acquired, including core, rock fragments, powdered cuttings and volatiles. There are many types of bits that are made in various shapes, with different cutting surfaces, flute configurations and dimensions, in addition to the material composition of its body and cutting edge surface. Examples of some of the bits are described in this section.

\subsection{1}

\section{Twist Drill Bits}

The most widely used and familiar bit in rotary drilling is the type called twist drill bit. This type of bits is used to drill holes in metal, rocks, plastic and wood. Examples of bits, including twist drill bits, are shown in Figure 1.10. The twist drill bit was invented in 1861 by Steven A. Morse, East Bridgewater, MA, USA, for which he received US Patent No. 38119 , dated April 7, 1863. The bit was originally produced by cutting two grooves on opposite sides of a metal rod and twisting it to create helical 


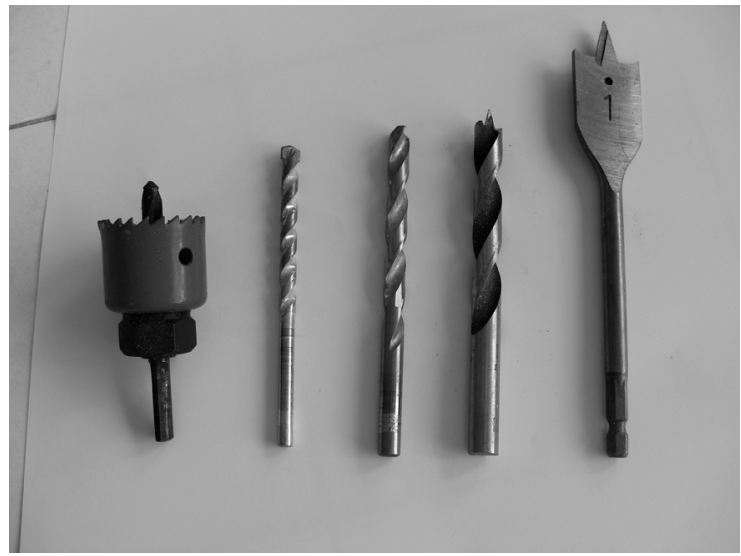

Figure 1.10 An assortment of drill bits including twist drill bits (the middle three).

flutes and therein lies the source of the bit name. Today, twist drill bits are produced by rotating a rod while cutting flutes via a grinding wheel. The performance of the bit is dictated by the geometry and shape of its cutting edges and the bit loses its effectiveness during use as its sharpness degrades.

The geometry of a twist drill bit is defined by its spiral or rate of twist, and also the pointing and lip angles, and these characteristics determine the drill performance. The spiral or rate of twist controls the rate of cuttings removal that can be optimized by a trade-off between competing desires for fast removal of cuttings and fast drilling speed. The optimum pointing angle is determined by the material that needs to be drilled, in which harder materials require a larger pointing angle, while softer materials require a steeper angle. This angle controls the bit walking and the bit chatter and also the shape of the drilled hole and the wear rate. The lip angle determines the amount of support provided to the cutting edge. A higher angle causes more aggressive cutting under a given axial load, but it can lead to binding, extensive wear and bit failure. The bit is also characterized by its length and diameter and the material being cut. The most commonly used cutting angle of twist drill bits is $118^{\circ}$. For very soft plastics a cutting angle that is close to vertical angle of about $90^{\circ}$ is used. On the other hand, a shallower angle in the range of $160^{\circ}$ is used to drill tough metals such as steel alloys. This type of bit requires a starter hole. To avoid binding and premature wear, it is necessary to maintain a proper drilling rate. A bit with no cutting angle is used to produce holes with a flat bottom, but these bits are very sensitive to changes in their lip angle and even a slight change can cause premature wear due to excessively fast cutting.

\section{4 .2}

\section{Gun Drill}

Gun drill bits (Figure 1.11) are used for drilling long, straight holes in metals, wood and some plastics and they carry this name because they are also used to produce 


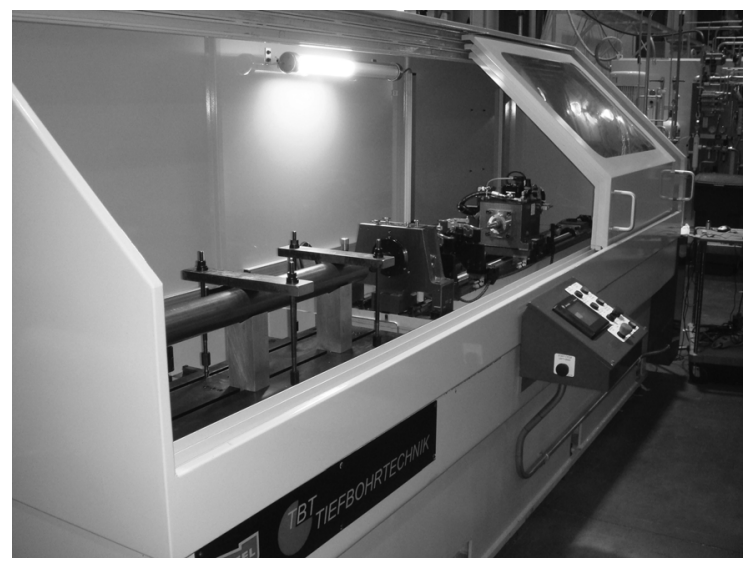

Figure 1.11 A photograph of a gun drilling machine (made by Nagel-TBT). Courtesy Nagel-TBT, USA.

gun barrels. Such bits create very straight and accurately sized holes as required for firearms. The bit has a hole through its core for the flow of a coolant, which may consist of a compressed air or a liquid. The coolant provides lubrication and cooling to the cutting edges and also acts as a carrier for removal of the cuttings. Generally, the bits are made with a carbide tip to allow drilling through harder materials while extending the life of the drill bit.

\subsection{3}

\section{Centering and Spotting Drill Bits}

Centering (Figure 1.12) and spotting bits are means of providing a starting hole for large-sized drill bits or to make a conical indentation in a surface. Drill walk or wander is prevented when drilling into an unprepared surface by first making an initiation hole. A spotting drill bit is designed with an angle that is the same as or greater than that of conventional bits to allow starting of a drill with a large bit with minimal stresses on the bit corners. This approach helps to minimize premature failure of the bit or damage to the borehole quality. Generally, solid-carbide drills are specifically designed to start their own hole. The use of a centering punch tool to produce a pilot hole is an alternative to the use of these types of bits for initiation of a drilled hole.

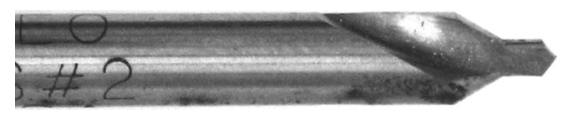

Figure 1.12 A photograph of a centering bit that is used to start holes for drilling with minimal drill walk. 


\subsection{4}

\section{Material Makeup of Bits}

The material that is used to fabricate a bit is critical to its performance, durability and cost, and also the application for which the bit is intended. Some of the materials that are used to make bits include soft low-carbon steel (for wood) and high-carbon steel that are hardened and tempered (for wood and metals). Excessive heating of the bit causes loss of the tempering and softening, leading to subsequent dulling of the cutting edge. Other materials that are used for higher speed drilling and hard materials drilling include tool steel, cobalt steel and tungsten carbide (due to its cost and brittleness it is used in small pieces that are screwed or brazed on to the tip of the bit). An example of a drill bit that is used for rocks and masonry utilizing a hardened blade for improved fracture is shown in Figure 1.13.

In certain applications, single diamond crystals are embedded into the tips of cutting tools. Diamond is the hardest known material and is used to produce tools that last much longer, but they are also much more expensive. A diamond crystal exhibits large strength anisotropy (i.e., it has hard and soft surfaces) and can in fact be very brittle when impacted at some angles (Denning, 1953). To deal with the diamond anisotropy, many diamonds are typically fused together to form a polycrystalline diamond. Polycrystalline diamonds are weaker on average than the hardest diamond surfaces, but they are much stronger than the weakest diamond surface. Naturally occurring polycrystalline diamonds are relatively rare and most of the polycrystalline diamonds used by industry are synthetic. Polycrystalline diamonds offer much higher toughness (i.e., resistance to brittle fracture) and hardness. When used as a cutter, a polycrystalline diamond is bonded to a tungsten carbide substrate layer and it is often called PDC (polycrystalline diamond compact). The abrasion resistance of the diamond layer removes the rock by shearing action, while the tungsten carbide layer provides mechanical support and impact resistance (Figure 1.14). Unfortunately, diamonds are not suitable for drilling ferrous materials such as steel. This is because when heated to temperatures as low as $500^{\circ} \mathrm{C}$, diamonds in the presence of certain metals such as iron or cobalt (e.g. in steels) tend to revert to graphite (which is the stable allotrope of carbon at atmospheric conditions) (Evans and Phaal, 1962). A less effective (but also lower cost) alternative to diamond is the artificial crystal cubic Boron Nitride.

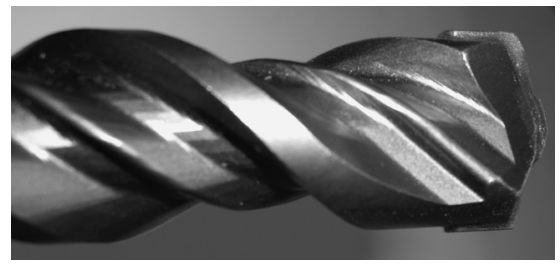

Figure 1.13 A photograph of a bit that is used in a hammer-rotary drill where a hardened blade is embedded into the end effector of the bit. 


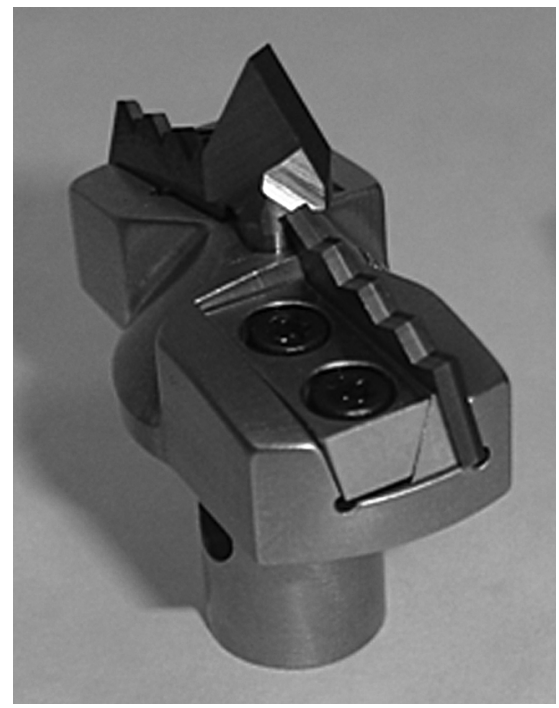

Figure 1.14 Example of a drill bit utilizing PDC for cutting rocks.

The serrated cutter is a PDC that consists of two layers:

polycrystalline diamond (dark layer) and tungsten carbide

(light layer).

Sometimes, to increase the wear resistance of the bit and its ability to drill hard materials, various coatings are applied, including black oxide (FeO), titanium nitride, titanium aluminum nitride, titanium carbon nitride TiCN) and zirconium nitride, and also diamond powder embedded in the surface of the bit.

\section{5}

\section{Application of Drilling Techniques}

\subsection{1}

\section{Geological Studies and Search for Resources}

Penetrating the ground and extracting samples for analysis are essential for many geological studies (Further details can be seen in Chapter 3). Such studies provide vital data in the search for resources (e.g., petroleum, gold, coal), to support construction plans prior to building roads and buildings and for purely scientific endeavors. The most valuable samples are in the form of continuous cores because they provide a graded historical picture of the subsurface, that is, stratigraphy of the subsurface. While searching for mineral resources, such cores can be used to assess the grade of minerals within the rock matrix and in turn determine the economic feasibility of a prospective mining operation. For geotechnical purposes, a carefully extracted sample from a core is used to assess the strength of the subsurface formation and thereby determine whether the ground is strong enough to support 
planned buildings and roads. Cores are also used to learn about the past history of our planet Earth. For example, ice cores acquired from the deepest parts of Antarctic ice sheets were determined to have age in the range of 800000 years. These ice cores are used to identify the chronology of previous ice ages and interglacial periods, to map out the history of variations in the Earth's climate and to sample the atmospheres that prevailed during those times.

\section{5 .2}

\section{Mining and Tunneling}

Mining and tunneling are two large-scale applications of excavation techniques that require powerful drilling tools. Mining is the extraction of valuable materials from natural sources by means of surface and underground mining tools. Initially, mining was simply carried out by picking up loose rocks and using them as tools to enhance the excavating capability of the human hands and fingers. Using hard rocks, humans were able to extract soft ores, but the eventual use of metals allowed humans to greatly increase their mining capability. Specifically, the use of iron, which began in the Iron Age during the second millennium BC, improved people's lives in many ways and led to the development of tools that were hard enough to drive into natural cracks in ores and force them apart. For many years fire was used to induce stresses in rocks and cause them to fracture and, as recently as the $1880 \mathrm{~s}$, this process was used by Japan for producing long tunnels. The most profound impact on mining was made around the late 1500 s through the use of gunpowder to create explosions. Initially, miners relied on cracks in rocks into which they inserted the gunpowder, but with time it was realized that using iron tools, it was possible to create deep holes for more effective explosions and excavations. The first documented use of drilling and blasting for mining was made in 1613 by Martin Weigel at Freiberg, Germany. The use of improved metallurgical processes to increase the strength and durability of the tools led to increasing improvements in the ability to drill and excavate.

In order to reduce the cost of labor and also remove humans from hazardous or often unpleasant environments, many drill rig manufacturers increasingly fund projects that seek to automate the entire drilling process. A good example of a fully autonomous rig is the Atlas Copco family of SmartRigs (Figure 1.15). The entire process of drilling starts with the prospective driller plotting out on a laptop computer the location, depth and inclination of the required holes. The operator then uploads the data into the drill rig computer and from this point onwards the operator/driller can observe the execution of the drill task from the safety of a comfortable cabin. The drill rig is equipped with all the sensors required to position the drill in a given location and to maintain the required penetration rate. In addition, the drilling telemetry, such as weight on bit, rotational speed and penetration rate, are recorded in real time; this is referred to as measurement while drilling (MWD). For open-cast mining, the data from MWD can be used to assess the strength of the formation and in turn assess the quantity of explosives that would be required for blasting coal or other mineral resources. 


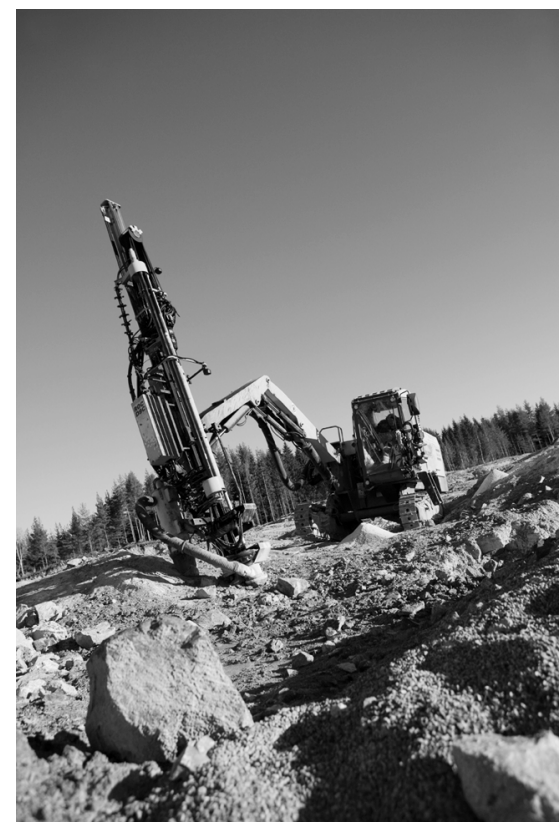

Figure 1.15 Atlas Copco SmartRig ROC D9C. The drill literally drills by itself. Courtesy Atlas Copco Rock Drills AB, Sweden.

\subsection{3}

\section{Petroleum and Gas Drilling and Exploration}

Most exploration drilling on Earth is intended to search for mineral resources and is motivated by financial return. Oil exploration is a relatively young field. Only late in the nineteenth century was it recognized that oil too, and not just coal, can be an important source of energy (Hunt, Philp and Kvenvolden, 2002). The resultant hunger for oil-based energy has accelerated the development and use of powerful drilling tools that can reach great depths both on the ground and underwater on the seafloor. Countries today are analogous to a living cell - they need energy to survive and petroleum products are expected to remain one of the most important sources of energy for at least several decades. Drilling for oil is attempted anywhere that oil may occur, including offshore drilling and even in schoolyards, as in the Los Angeles area. It is evident that new drilling technologies will have to be invented to reach deeper oil reservoirs to satisfy this hunger for energy. Therefore, petroleum drilling and exploration are expected to drive significant advances in drilling technologies.

Traditionally, oil and gas wells have been drilled via a string of rotatable rigid steel pipe sections with a drill bit attached to the end. The process of changing bits involves pulling out and unscrewing each section of the drill string and then screwing it back while lowering into the borehole and this can take hours or even days to perform for deep holes. In recent years, an alternative has emerged that utilizes coiled tubing consisting of continuous flexible tube that is stored on a reel and may be as long as 
22 1 Drills as Tools for Media Penetration and Sampling

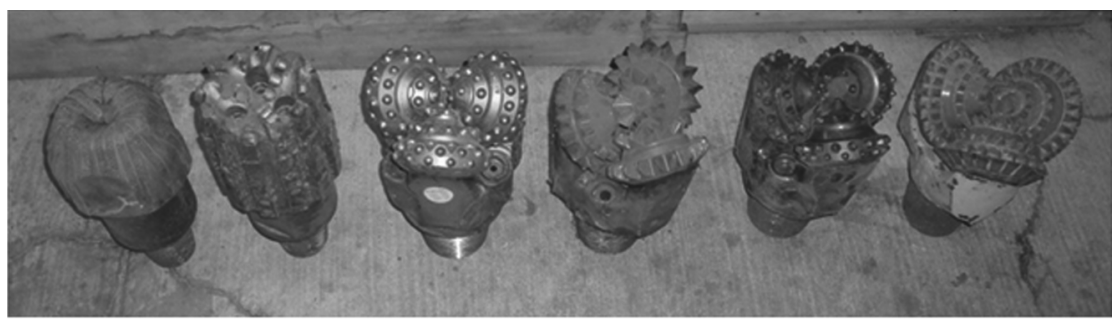

Figure 1.16 Petroleum drill bits are produced in various shapes depending on the formation that needs to be penetrated.

From left to right: surface set diamond bit; PDC bit; tungsten carbide tricone bit; and tricone milled tooth bit with different teeth shapes and sizes. (Please find a color version of this figure on the color plates.)

$3 \mathrm{~km}(10000 \mathrm{ft})$ or more. A hydraulic motor and a drill bit is attached to the coiled tubing end and it drills boreholes while fluid is forced through the tubing to drive the motor and rotate the drill bit. The drill bit and the hydraulic motor are lowered into the borehole while unspooling the coiled tubing. This method allows raising and lowering of the drill in the borehole at rates that are as much as 10 times faster than it is possible with conventional rotary drilling techniques.

The range of drill bits used in petroleum well drilling has been increased significantly in recent years as a result of development of new types of cutter elements such as polycrystalline diamond and thermally stable polycrystalline diamond. The drill operator has an option of selecting which bit to use depending on the type of formation and the hardness that each bit can handle. Thus, there are soft rock bits for soft formations such as shale, soft limestone and sandstone, medium hard rock bits for formations such limestone and dolomites and very hard formation rock bits for formations such as hard shale, mudstones, and also hard and abrasive formations. Figure 1.16 shows six types of drill bits which are most commonly used in well drilling and they include surface set diamond bits, polycrystalline diamond compact (PDC) bits, tungsten carbide inserts (TCI), tricone bits and three tricone milled tooth (MT) bits with different shapes of teeth. The tricone bits were invented in 1933 by engineers at Hughes Company. The tricone bits are named for their cones that rotate around the bit axis. There are also dual and single cone bits. The predominant type of drill bits in the petroleum industry are tricone and PDC bits. PDC bits are much more expensive, costing sometimes in excess of $\$ 100000$. The bits shown in Figure 1.16 are as follows:

- The surface set diamond bit consists of single diamonds set in a metal matrix and they are used to drill in very hard formations.

- The PDC bit consists of PDC buttons that shear the rock and are used to drill soft rocks.

- Roller cone bits consist of wheels with teeth which essentially turn as the bit is rotated. The teeth apply pressure on to the rock at a level that exceeds the strength of the rock 
and in turn break up the rock. The tricone bits with shorter, closely arranged teeth with TCIs are used for harder formations. These bits have longer (more protruding) and widely arranged teeth and are used for softer formations. Generally, bits with shorter teeth penetrate the rock much slower than bits with longer teeth.

\subsection{4}

\section{Ocean and Seafloor Drilling}

Improvements in drilling capability have enabled researchers and engineers to take on ever-increasing challenges, including drilling from ships that dynamically hold their position above the seabed (Further details can be seen in Chapter 5). Today, drilling in water depths of over $2000 \mathrm{~m}$ is possible while penetrating to depths of many thousands of meters through the subsurface underneath. The US National Science Foundation (NSF) is one of the leading government agencies that funds such drilling projects with the objective of improving the understanding of the origins of devastating earthquakes, studying the Earth's history through coring of unstable thick sediment sections and investigating the fundamentals of ocean crust formation. Oil exploration companies are dominant in the efforts to drill under the ocean water and the significant increase in demand for energy is leading to numerous platforms being placed at various sea locations of the world, including the Gulf of Mexico and the North Sea.

\subsection{5}

\section{Planetary Drilling and Sampling}

Extraterrestrial drilling dates back to the 1970s, when Apollo 15 astronauts (Commander David Scott and Lunar Module Pilot James Irwin) drilled a hole on the Moon to a depth of $2.4 \mathrm{~m}$ and recovered a continuous core sample. The drill, called the Apollo Lunar Surface Drill (ALSD), was a $500 \mathrm{~W}$ rotary-percussive drill with a coring bit capable of acquiring a continuous $2 \mathrm{~cm}$ diameter core and an auger for carrying cuttings to the surface (Figure 1.17).

Increasingly, the objectives of planetary exploration missions involve in situ sampling and analysis, but the challenges are significantly more complex than most scientific drilling on Earth (Briggs and Gross, 2002; Zacny et al., 2008; Chapter 6 of this book). Drilling a hole without astronaut control on a distant planetary body can be compared to driving a planetary rover in complete darkness with no view of the road ahead. The reason is that there is only limited information available from the drilling process to indicate what is being drilled. Further, the task must be accomplished within the challenging overall mission limits on mass, power and size. Because extraterrestrial drilling offers the prospect of major scientific discoveries, there is ample motivation and incentive for the planetary science and engineering community to tackle the daunting development tasks.

One of the major goals of the planetary exploration programs of the National Aeronautics and Space Administration (NASA) is to search for evidence that life may have developed elsewhere in our solar system - in particular on Mars. The 


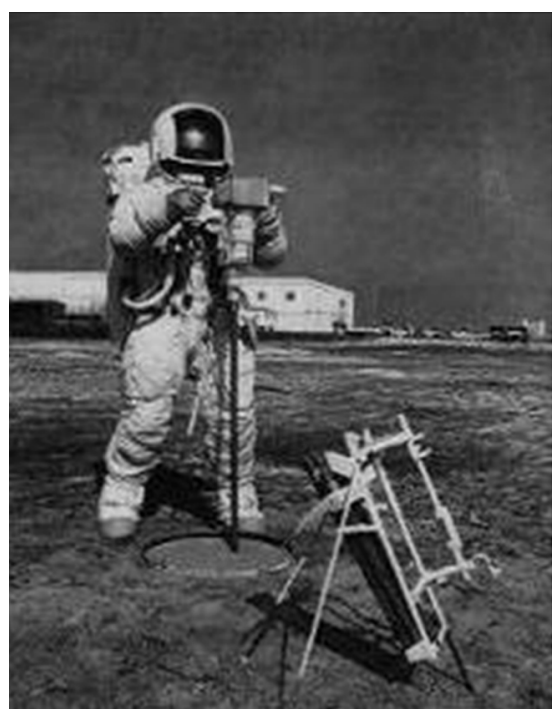

Figure 1.17 Apollo lunar surface drill being tested at the Kennedy Space Center. The handle, battery, power head and drill stems are visible. The drill stem rack is in the foreground with a set of stem sections and a wrench on the right. Courtesy NASA (photograph S70-29673).

subsurface is a more likely venue for such evidence - if it exists. Samples acquired from the Martian subsurface and analyzed at the site or returned for analysis back to Earth represent our best opportunity to resolve this long-standing question. More generally, through an assessment of the stratigraphy of acquired samples, it becomes possible to peer back directly into the often complex geological history of a planet. The Apollo experience indicates how powerful such sample analyses can be. Further, such studies can aid future human exploration by locating resources that can be used to "live off the land" by means of in situ resource utilization. Similarly, knowledge of the mechanical properties of the soil would be necessary for construction of habitats and assessment of the trafficability for a human outpost. Data from drill telemetry can also be helpful for material characterization and may be used to identify changes in stratigraphy in order to identify regions that have scientific value for further investigation.

The geological record of a planet is created by material emplacement and thus the stratigraphic record is one in which older events are buried beneath more recently emplaced material. On Earth, where erosion rates are high, material accumulates rapidly; resurfacing is a constant process and most surfaces are geologically young. On Mars, however, without liquid water to provide high erosion rates, the average resurfacing rate is very low (Herkenhoff, Plaut and Nowicki, 1997; Golombek and Bridges, 2000). This extremely slow resurfacing is the reason why ancient features, such as rivers and lake-beds, can be easily seen and reached. Therefore, on such planets as Mars, one may obtain important information using even relatively shallow 
drilling. In locations where active resurfacing processes occur, such as the ebb and flow of annual snowfall at the Martian polar caps, accumulation rates of entrained dust can be much higher. This is most likely the source of the polar layered terrains and it has been hypothesized that they may contain evidence of former primitive life (McKay, 1997; Cockell and Barlow, 2002; Smith and McKay, 2005).

\subsection{6 \\ Ice Drilling}

Drilling ice, particularly at very low temperatures, can be a challenging task. In addition to the associated hardness of the medium, there is a concern that melted ice can turn into frozen water that can jam the bit upon refreezing. The topic of ice drilling is discussed in greater detail in Chapter 4. Using the Jet Propulsion Laboratory (JPL) ultrasonic/sonic "gopher", the frozen layer of ice on Lake Vida 'Antarctica' was drilled to a depth of $176 \mathrm{~cm}$ using the percussive mechanism of the ultrasonic/sonic driller/corer (USDC) (Bar-Cohen et al., 2007). This drill used a piezoelectric actuator to excite resonant hammering action. Figure 1.18 shows photographs of the drilling site and the drilled borehole with the gopher inside.

\subsection{7}

\section{Dental Drills}

The drill is one of the most important tools that are used by dentists (Black, 1908; Simonsen, 1989; Jedynakiewicz, 1992; Christensen, 2006). They are applied to remove decayed tooth material prior to the insertion of a dental filling (Figure 1.19). The introduction of the dental drill revolutionized the field of dentistry and primitive drills were already in use as early as the eighteenth century. In 1868, the American dentist George F. Green introduced the first dental drill that was pneumatically powered via a pedal and bellows. Later, in 1875, he patented an electrified dental drill using an electromagnetic motor. Evolutionary improvements in performance, reliability and comfort of patients continued to be made at the early part of the twentieth

(a)

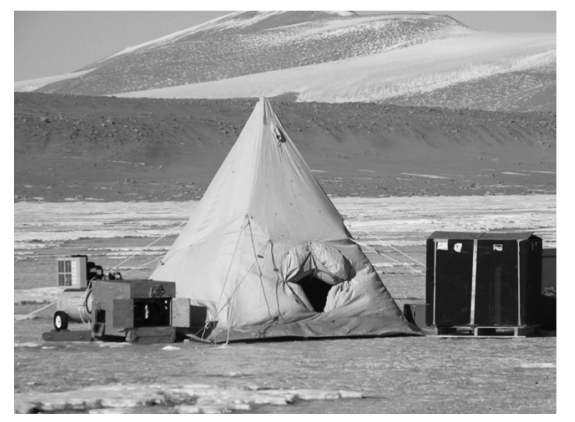

(b)

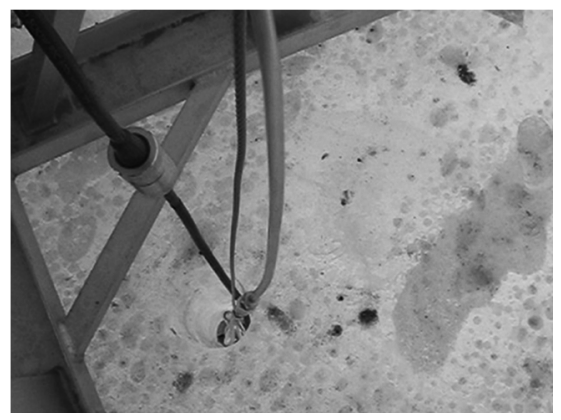

Figure 1.18 Drilling ice at Lake Vida, Antarctica, using the ultrasonic/sonic "gopher". 


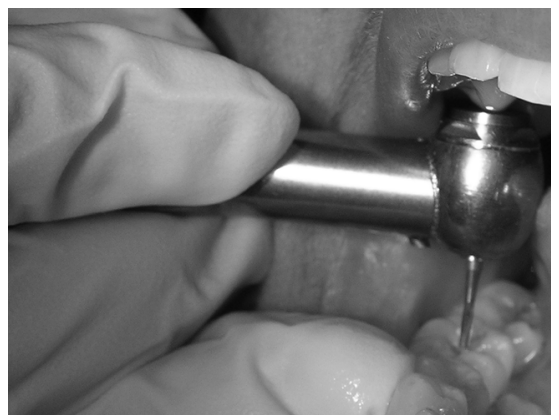

Figure 1.19 Dental drills are used to remove decayed tooth material. Courtesy Alan Grant, Dentist, Long Beach, CA.

century. These improvements led to the modern dental drills that work more quickly and accurately than ever before, with less pain for the patient. In 1911, high-speed drills were introduced, followed by a significant enhancement in 1953 using an air turbine motor that powers the modern dental drills. These drills convert highly pressurized air into mechanical energy and make the drill bit spin at speeds of thousands of rpm.

The requirements for the handpiece, which holds the rotating bit and supports the air turbine, include being lightweight and corrosion resistant. Air and water are applied for cleaning, cooling and operation of the turbine. The handpiece consists of ceramic or metal bearings and a central spindle with a chuck to hold the bit. The air is fed via a fine tube into the turbine that is driven at a pressure of about $35 \mathrm{psi}$. Without a load, the bit can spin at speeds of about $325000 \mathrm{rpm}$, and some dental drills even have significantly higher speeds. Upon application to the surface of a tooth, the torque load reduces the speed to $80000 \mathrm{rpm}$. A fine spray mist of air and water is used to cool the bit and remove the cuttings. The handpiece, internal bearings and the lubricant need to sustain an extensive number of operation cycles in addition to repeated sterilization in an autoclave.

The bits, which are called "burs" in dentistry terminology, are designed in a great variety of shapes as required by specific applications. A typical bur has a diameter of $0.8-1 \mathrm{~mm}$ with a cutting surface section that is about $4 \mathrm{~mm}$ long. They are generally made of steel with a tungsten carbide coating or entirely of tungsten carbide, and sometimes they are coated with diamond or other abrasive material. The tooth enamel, which is the hardest material in the human body, is an exterior crystalline structure made of calcium hydroxyapatite. To avoid "drill-walk" on the surface of the tooth, which can cause extensive damage to the enamel, the bur is applied in light strokes to cause microscopic milling of the surface without stalling the bur. The dentist's task is difficult given the confined operation space of the mouth, the related obstruction to the view, the limited light and the presence of various fluids that also include the grinding debris. The challenges are compounded by the fact that sometimes the dentist is drilling while looking through a mirror and working backwards. 


\section{Conclusion}

Drilling technology has benefited from advances in materials and other technologies and has contributed significantly to mankind's quest to explore. The enormous capabilities of drilling that emerged in the last 100 years have helped to build the infrastructure of our cities, including the freeways, tunnels, sewage and water systems and other utilities that we take for granted. Beginning on the Moon, drilling technology has also demonstrated the ability to explore the subsurface of other planetary bodies in the Universe - something that until recently would have seemed to lie beyond the wildest dreams of humans.

The technology needed to penetrate the subsurface on Earth is a mature field with wide scope and a multitude of specialized tools. The desire to make drilling more efficient provides the incentive to develop new related physical models, analytical approaches and innovative end-effector systems. The results are expected to lead to longer-lasting drills, improved penetration rates and much higher levels of autonomy. In the petroleum drilling industry, the desire to automate the process is driven by the need for manpower reduction and increased profitability, whereas for extraterrestrial drilling, the effort is motivated by the need to address the long communication delays and mass and power limitations.

As always, the issues of safety, power and cost will dictate selection of methods for any application. As drilling technology continues to make significant progress, the challenges that were once considered to be difficult or impossible to address, are expected to be solved. These challenges include: the ability to drill to greater depths and in inaccessible locations; operation at very high or extremely low temperatures; drilling in hazardous or environmentally sensitive areas; drilling under yet greater depths of ocean water; and, in the coming decades, drilling in remote locations in the solar system.

\section{Acknowledgments}

Some of the research reported in this Chapter was conducted at the JPL, California Institute of Technology and Honeybee Robotics Spacecraft Mechanisms Corporation under a contract with NASA. The authors would like to express their appreciation to the many scientists and engineers who contributed to the development of the reported technologies and who are from the authors' affiliations and other partner organizations. The authors would like to thank Nathan Bridges, JPL, for the information relating to the detection of life on Mars and other planetary bodies. Also, they would like to express their appreciation for the contributions of Donald Rapp, Independent Contractor (http://home.earthlink.net/ drdrapp/), Geoffrey A. Briggs, NASA Ames, and Greg S. Mungas, JPL, for reviewing this chapter and for providing valuable technical comments and suggestions. The authors would like to thank Alan Grant, DDS, Long Beach, CA, for his input to the description of dental drilling and for providing the related photograph. 
28 1 Drills as Tools for Media Penetration and Sampling

\section{References}

Bao, X., Bar-Cohen, Y., Chang, Z., Dolgin, B.P., Sherrit, S., Pal, D.S., Du, S. and Peterson, T. (2003) Modeling and computer simulation of ultrasonic/sonic driller/corer (USDC). IEEE Transaction on Ultrasonics, Ferroelectrics and Frequency Control (UFFC), 50 (9), 1147-1160.

Bar-Cohen, Y. (2005) Biomimetics - Biologically Inspired Technologies, CRC Press, Boca Raton, FL, pp. 1-527.

Bar-Cohen, Y., Sherrit, S., Bao, X., Badescu, M., Aldrich, J. and Chang, Z. (2007) Ultrasonic/ Sonic Driller/Corer (USDC) as a subsurface sampler and sensors platform for planetary exploration applications. Proceedings of the NASA Science Technology Conference (NSTC-07), University of Maryland Conference Center, June 19-21.

Black, G.V. (1908) Operative Dentistry, 9th edn, Vol. II, Medico-Dental Publishing Company, Chicago, IL, pp. 110-116.

Briggs, G. and Gross, A. (2002) Technical Challenges of Drilling on Mars. American Institute of Aeronautics and Astronautics, Report No. 0469.

Burleson, C.W. (1998) Deep Challenge: Our Quest for Energy Beneath the Sea, Gulf Professional Publishing.

Christensen, G.J. (2006) The 'new' operative dentistry. Journal of American Dentists Association, 137 (4), 531-533.

Clark, G.B. (1987) Principles of Rock Fragmentation, John Wiley \& Sons, Inc., New York

Cockell, C.S. and Barlow, N.G. (2002) Impact excavation and the search for subsurface life on mars. ICARUS, 155 (2), 340-349.

Dansgaard, W. (2004) Frozen Annals Greenland Ice Sheet Research, The Niels Bohr Institute, University of Copenhagen.

Decker, A.G. and Black, S.D. (1917) Electrically-driven tool, US Patent No. $1,245,860$.

Decker, A.G. and Black, S.D. (1961) http:// www.blackanddecker.co.uk/about/ highlights/ Accessed 9 January 2008.
Denning, R.M. (1953) Directional grinding hardness in diamond. American Mineralogist, 38, 108-117.

De Villiers, M. (2001) Water: The Fate of Our Most Precious Resource, Houghton Mifflin Company.

Encyclopedia Britannica (1986) Encyclopedia Britannica, Vol. 4, Encyclopedia Britannica, Chicago, IL, p. 227.

Evans, S. and Phaal, C. (1962) Proceedings of the 5th Conference on Carbon, Pergamon Press, New York.

Golombek, M.P. and Bridges, N.T. (2000) Erosion rates on Mars and implications for climate change: constraints from the Pathfinder landing site. Journal of Geophysical Research, 105 (E1), 1841-1854.

Heiken, G. and Jones, E. (2007) On the Moon: The Apollo Journals, Springer.

Herkenhoff, K., Plaut, J.J. and Nowicki, S.A. (1997) Surface age and resurfacing rate of the north polar layered terrain on Mars, LPSC, Abstract 1575.

Hunt, J.M., Philp, R.P. and Kvenvolden, K.A. (2002) Early developments in petroleum geochemistry. Organic Geochemistry, 33 (9), 1025-1052.

Jedynakiewicz, N. (1992) A Practical Guide to Technology in Dentistry, Wolfe Publishing.

Jerby, E., Dikhtyar, V., Aktushev, O. and Grosglick, U. (2002) The microwave drill. Science, 298 (5593), 587-589.

Just, G.D. (1963) The Jet piercing process. Quarry Managers' Journal, Institute of Quarrying Transactions, 219-226.

Rao Karanam, U.M. and Misra, B. (1998) Principles of Rock Drilling, Balkema Press, Rotterdam, pp. 1-265.

Kola (1989) http://en.wikipedia.org/wiki/ Kola_Superdeep_Borehole\#_note-0. Accessed 9 January 2008.

Lee, T.H., Linden, H.R., Dreyfus, D.A. and Vasko, T. (1988) The Methane Age, Springer, New York.

Maurer, W.C. (1968) Novel Drilling Techniques, Pergamon Press, New York. 
Maurer, W.C. (1980) Advanced Drilling

Techniques, Petroleum Publishing Company,

Tulsa, OK.

McGee, E. (1955) New down-hole tool. Oil and Gas Journal, 54, 67.

McKay, C. (1997) The search for life on Mars. Origins of Life and Evolution of Biosphere, 27 (1-3), 263-289.

Mining Magazine (2006) Breaking versus blasting. Mining Magazine, 194 (3), March, 14-17.

Ostrovskii, N.P. (1960) Deep-Hole Drilling with Explosives, Gostoptekhia 'dat, Moscow (Translated by Consultants Bureau Enterprises Inc., New York).

Peele, R. (1920) Compressed Air Plant: the Production, Transmission and Use of Compressed Air, 4th edn, John Wiley \& Sons, Inc., New York.

Rapp, D. (2007) Human Missions to Mars: Enabling Technologies for Exploring the Red Planet, Springer/Praxis Publishing, 3540729389, Appendix C, Water on Mars.

Ready, J.F. (1997) Industrial Applications of Lasers, 2nd edn, Academic Press, New York.

Simonsen, R. (1989) Dentistry in the 21st Century - A Global Perspective, Quintessence Publishing Co., Hanover Park, IL.

Smith, H. and McKay, C. (2005) Drilling in ancient permafrost on Mars for evidence of a second genesis of life. Planetary and Space Science, 12, 1302-1308.

Smith, M., Cardell, G., Kowalczyk, R. and Hecht, M.H. (2006) The Chronos thermal drill and sample handling technology. 4th International Conference on Mars Polar Science and Exploration, Davos, Switzerland, 2-6 October, Abstract 8095.

Wyllie, D.C. (1999) Foundations on Rock, 2nd edn, Taylor and Francis Press, Boca Raton, FL, pp. 345-347.

Zacny, K., Bar-Cohen, Y., Boucher, D., Brennan, M., Briggs, G., Cooper, G., Davis, K., Dolgin, B., Glaser, D., Glass, B., Gorevan, S., Guerrero, J., Paulsen, G., Stanley, S. and Stoker, C. (2008) Drilling systems for extraterrestrial subsurface exploration. Journal of Astrobiology, 8 (3), 665-706.

\section{Internet Links}

Coal mining: http://www.britannica.com/eb/ article-81659/coal-mining http://www.allproducts.com/search/ products/pM01012714.shtml.

Compressed air drills: http://www.gmm.gu se/groups/pedersen/popDetail.php? ID $=8$

http://books.nap.edu/openbook.php? record_id $=11558 \&$ page $=129$

http://www.iodp.org/images/stories/ downloads/sd5_07.pdf http://www.iodp-usio.org/http://www.cbc. ca/news/background/mining/.

Drills and drill bits: https://edit.britannica. com/getEditableToc?tocId $=81255$

http://en.wikipedia.org/wiki/Drill http://www.thefreedictionary.com/drill http://www.britannica.com/ebc/article9363107.

Explosive drilling: http://ntlsearch.bts.gov/ tris/record/tris/00051928.html http://cedb.asce.org/cgi/WWWdisplay.cgi? 9200021.

Gun drills: http://www.gundrillingsolutions. com/Pages/gundrl.html.

History of drilling: http://www. americanheritage.com/articles/magazine/ it/1999/1/1999_1_56.shtml http://homepage.ntlworld.com/leslie. foster/drilling_history.htm http://www.mining-journal.com/ mining_magazine/pdf/MM0603scr.pdf http://www.americanheritage.com/articles/ magazine/it/1999/1/1999_1_56.shtml.

Jackhammer: http://en.wikipedia.org/wiki/ Jack_hammer.

Mining technologies: http://www.miningtechnology.com/contractors/drilling/gallery. html.

Rock excavation tools: http://books.nap.edu/ openbook.php?record_id $=2349 \&$ page $=63$.

Rock drilling: http://www.americanheritage. com/articles/magazine/it/1999/1/ 1999_1_56.shtml.

Tool materials: http://arjournals. annualreviews.org/doi/pdf/10.1146/ annurev.ms.09.080179.000323? cookieSet $=1$. 
